\title{
Effectiveness of oral health promotion in changing behaviour is not proven
}

\author{
Kay E J, Locker D. Effectiveness of Oral Health Promotion: a review. Health Education Authority 1997
}

\begin{abstract}
Objective To determine whether oral health promotion brought about any improvement in oral health. Oral health was not limited to clinical measures of knowledge, attitudes and behaviour; the validity of measures to assess these outcomes was also assessed.
\end{abstract}

Data sources Studies were identified from Medline, E.Med, Cancerlit, Dissertation Abstracts Outline, government documents listings, Current research in Britain, Health service abstracts, reference lists of retrieved articles, personal files of topic experts as well as a hand search of 96 relevant journals. Articles were published in English or with English abstracts between 1980-1995.

Study selection Three types of study design were included: randomised controlled trials, quasi-experimental studies and single group studies. Studies were excluded if no measure of outcome was reported (i.e. descriptive studies).

Data abstraction Each paper identified was classified by study design, target population, setting, outcome measure and follow-up period. A list of 21 items was used to assess quality. Quantitative and qualitative data synthesis was carried out where appropriate.

Main results 192 papers were retrieved: 28 descriptive or theoretical, 1 case study, 36 randomised controlled trials, 80 quasiexperimental and 33 single group studies.

Interventions aimed at reducing caries ( 7 RCTs) Most studies involved use of some form of fluoride application therefore making it difficult to measure any educational effect.

Interventions aimed at improving oral hygiene ( 23 RCTs) Not all measured plaque levels in same way. Mean effect in those studies using plaque index was a 0.316 reduction ( $95 \%$ CI $0.063-0.695)$.
As confidence interval crosses zero this means that oral hygiene is not reliably improved by interventions identified in studies. Interventions aimed at improving gingival health ( 5 RCTs) Many different indices used to record gingival health. Evidence suggests that if plaque levels are reduced some improvement in gingival health can be expected.

Interventions aimed at improving knowledge, attitudes, beliefs and behaviours ( 7 RCTs). Due to different ad hoc knowledge scales used it is difficult to compare studies. Studies suggest simple provision of information improves knowledge, and that few differences in behaviour could be attributed to knowledge.

Interventions aimed at altering sugar intake ( 1 RCT) This study showed a clear increase in recommendations for sugar-free preparations from pharmacists.

\section{Conclusions and recommendatiops}

Despite hundreds of studies involving thousands of individuals, we know remarkable little about how best to promote oral health. Sustained use of fluoride reduces caries and good oral hygiene promotes gum health and appears to be related self-esteem. Daily thorough brushing with fluoride toothpaste is an oral health behaviour and is an achievable goal. Manipulation of sugar consumption or other means of promoting oral health has not be proven to be effective. The review also recommended a series of actions for purchasers, policy makers and research.

Address E J Kay, Oral Health Development Department,
University of Manchester, Manchester M15 6FH, UK

Funding: Commissioned by the Health Education Authority, which is funded by the Department of Health. the methodologies but also the findings of these reviews. The HEA review makes mention of the HPW report and points out the differences, in two main areas. Firstly, they conclude that individually tailored programmes are no more beneficial than simple instructions in improving oral health. Secondly, they conclude that there is insufficient evidence tat increasing knowledge leads to behaviour change. The degree to which these differing conclusions are a consequence of the identified deficiencies in research quality and quantity is difficult to estimate.

The findings of the reviews are aimed principally at purchasers and those concerned with oral health strategies, but there is a great deal of interest to those involved in delivering oral health promotion in all areas of dental practice. The maxim that the lack of evidence is not the same as lack of effectiveness should be borne in mind and the HEA commentary urges investment in evaluation. However, the findings support the view that traditional dental health education is not shown to be effective and purchasing decisions in the future will reflect this.

These decisions are likely to be taken jointly with providers and there is a great deal in these reviews to help with the planning and evaluation of oral health promotion interventions in local areas. The HPW document particularly has useful summaries of study design, a classification of outcome measures and a guide to selecting reliable papers, cross reference to providers and locations by interventions. The evidence of effectiveness for interventions that could be located in general dental practice is helpful in supporting the role of the dental practice team in health promotion.

These reviews have pointed out the very real deficiencies in the literature on the evaluation of oral health promotion and should do much to stimulate further research and improvements in methodologies both in study design and in the evaluation of fieldwork in local dental services.

John R Green

Consultant in Déntal Public Health, Lincolnshire Health Authority, Lincoln, UK 